\title{
Helicity Generation and Signature in Solar Atmosphere
}

\author{
A. A. Pevtsov
}

National Solar Observatory, Sunspot, NM 88349, U.S.A.

\begin{abstract}
To fully understand the origin, evolution and topology of solar magnetic fields, one should comprehend their magnetic helicity. Observationally, non-zero helicity reveals itself in the patterns of electric currents inside active regions, superpenumbral sunspot whirls, the shape of coronal loops and the fine structure of chromospheric filaments. Some patterns may bear information about deep sub-photospheric processes (e.g., dynamo, turbulent convection). Others may originate at or near the photosphere. This presentation reviews the observations of magnetic and current helicity on the Sun, discusses the possible mechanisms of helicity generation, and compares them with the observations.
\end{abstract}

\section{Observations of Magnetic and Current Helicity}

Magnetic helicity $\left(\mathrm{H}_{m}\right)$ plays a fundamental role in understanding the origin, evolution and topology of solar magnetic fields on different spatial and temporal scales (for review, see individual papers in Brown, Canfield, \& Pevtsov 1999). $\mathrm{H}_{m}$ is a product of magnetic induction $\mathbf{B}$ and the vector potential $\mathbf{A}$ integrated over a closed volume $(\mathbf{n} \cdot \mathbf{B}=0$ on the volume boundary). Typically, the magnetic fields are observed in a single level in solar atmosphere (photosphere or chromosphere), and hence, $\mathbf{A}$ cannot be computed in a general case. However, the sign of helicity can be determined from existing observations. For example, one can use the $\alpha$ coefficient of linear force free field $\nabla \times B=\alpha B$ (Seehafer 1990; Pevtsov, Canfield, \& Metcalf 1995) or the vertical component of current helicity density $h_{c}=B_{z} \cdot(\nabla \times B)_{z}$ (Abramenko, Wang, \& Yurchishin 1997; Bao \& Zhang 1998). In addition, the sign of helicity can be inferred from the curvature of superpenumbral filaments (Hale 1927), the shape of coronal sigmoids (Rust \& Kumar 1996), the skew of coronal arcades (Martin \& McAllister 1996), or the orientation of the barbs in the chromospheric filaments (Martin, Bilimoria, \& Tracadas 1994). One can also determine the helicity of photospheric field relative to the potential field (Berger \& Field 1984; Chae 2001; Démoulin et al. 2002; Kusano et al. 2002).

The independent studies established following properties of helicity:

- Solar magnetic fields exhibit a tendency for negative (positive) helicity in the northern (southern) hemisphere. However, the latitudinal dependence shows significant scatter (Figure 1).

- The hemispheric rule is independent of solar cycle (Hale 1927; Martin et al. 1994; Pevtsov, Canfield, \& Latushko 2001). However, there were reports that the rule may not hold in some phases of solar cycle (Sakurai \& Hagino 2003). 


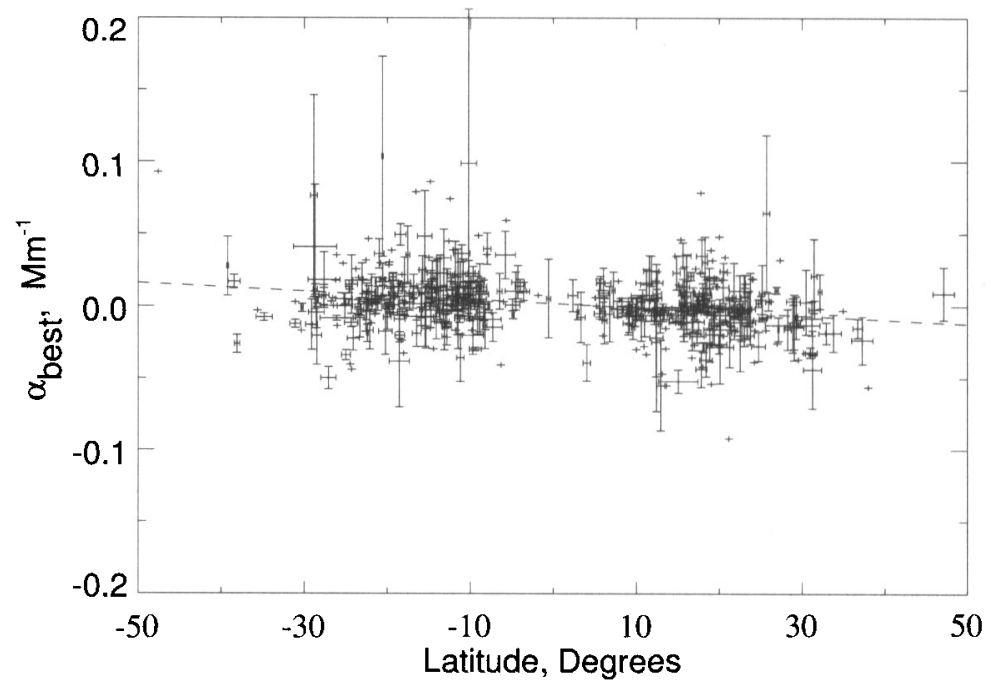

Figure 1. Latitudinal dependence of $\alpha$ for 466 active regions observed from 1998-2000 by the Haleakala Stokes Polarimeter (HSP, Mickey 1985). The dashed line is a least-square linear best fit.

- Magnetic flux emerges with non-zero twist (Leka et al. 1996; PortierFozzani et al. 2001; Grigoryev \& Ermakova 2002).

- Relative helicity of a typical active region is about $10^{43} \mathrm{Mx}^{2}$ (Updike \& Pevtsov 2003). In a large, CME-productive region coronal mass ejections may remove as much as $3.6 \times 10^{43} \mathrm{Mx}^{2}$ over a lifetime of the region (Démoulin et al. 2002).

\section{Origin of Helicity}

The hemispheric helicity rule may result from the differential rotation, action of the Coriolis force on a flux tube rising through the convection zone, subsurface dynamo, and turbulence-magnetic field interaction in the convection zone.

DeVore (2000) showed that the surface differential rotation may generate sufficient amount of helicity over a lifetime of a small active region. On the other hand, Chae (2001) and Moon et al. (2002) found that the contribution of the photospheric local horizontal motions may significantly exceed the contribution of the differential rotation. Démoulin et al. (2002) concluded that the differential rotation cannot account for helicity lost due to CMEs.

The Coriolis force acting on plasma flowing in the rising and expanding magnetic flux tube distorts the shape of the tube. Above the photosphere, the tube appears as a bipolar region tilted relative to the equator (Joy's law). In addition to tilt, the same action will twist the magnetic field inside the tube. If the magnetic helicity of active regions was created by this mechanism, the twist and tilt should be in negative correlation (the sign of correlation depends on the 
definition of sign of tilt). Indeed, Tian et al. (2001) found a negative correlation between the active regions' tilt and twist. On the other hand, Canfield \& Pevtsov (1998), and Sakurai \& Hagino (2003) concluded that a correlation between tilt and twist cannot be explained by the action of the Coriolis force.

Longcope, Fisher, \& Pevtsov (1998) suggested that the interaction between magnetic flux tubes and turbulent convection in the convection zone ( $\Sigma$-effect) may explain the weak hemispheric preference and significant scatter of the helicity rule. Longcope et al. (1999) showed that a contribution of the $\Sigma$-effect is comparable with the observed helicity of active regions. The estimated contribution of the overshoot-region and and mean-field dynamos (Longcope et al. 1999; Seehafer et al. 2003) is at least on the order of a magnitude smaller.

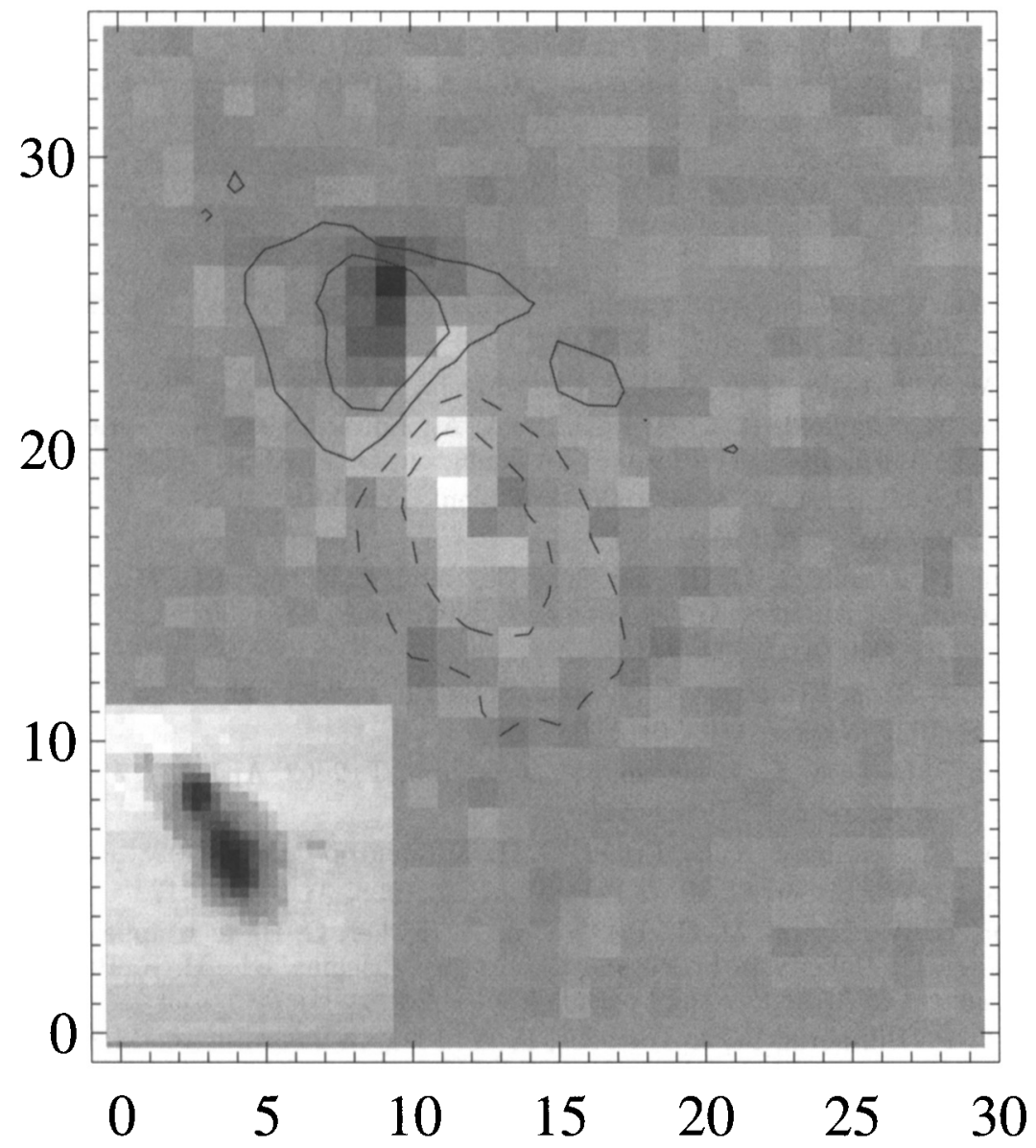

Figure 2. Electric currents (background) and vertical field (contours) of $\delta$-spots NOAA 6619 . Insert shows the white-light image.

The $\delta$-spots provide an additional support for the sub-photospheric origin of helicity. Upon emergence, these regions exhibit significant shear and strong electric currents. In a typical (not $\delta$-) sunspot both positive and negative currents 
are usually present inside a single polarity field (e.g., umbra). In the $\delta$-spots, however, the currents show strong polarity dependence. For example, in $\delta$-spot shown on Figure 2, the currents flow up in the negative polarity field, and they flow down in the positive polarity. This topology supports the model of a $\delta$-spot as significantly distorted (and perhaps kinked) flux tube (Linton et al. 1998). Using HSP vector magnetograms of three large $\delta$-spots, we computed the correlation between the vertical magnetic flux $\mathrm{B}_{z}$ and the vertical component of electric current density $\mathrm{J}_{z}$. In all three cases, the $\mathrm{J}_{z}$ and $\mathrm{B}_{z}$ are strongly correlated: $\tau$ $=-0.66 \pm 0.07$ (NOAA AR 5747), $-0.58 \pm 0.17$ (NOAA AR 6619), and -0.60 \pm 0.05 (NOAA AR 6659). This significant correlation indicates that in $\delta$-spots electric currents are confined to the corresponding polarities (similar to Figure 2). The NOAA 6659 was the second rotation of NOAA 6619 . However, the magnetic field was more twisted in NOAA 6619 , i.e., $\alpha=(-3.20 \pm 0.36) \times 10^{-8}$ $\mathrm{m}^{-1}$ (NOAA 6619) $\alpha=(-1.87 \pm 0.75) \times 10^{-8} \mathrm{~m}^{-1}$ (NOAA 6659). Thus, the evolution of the twist in this $\delta$-spot (NOAA 6619-6659) does not support the differential rotation as the source of helicity.

\section{References}

Abramenko, V. I., Wang, T. \& Yurchishin, V. B. 1997, Solar Phys., 174, 291

Bao S. \& Zhang, H. 1998, ApJ, 496, L43

Berger, M. A. \& Field, G. B. 1984, J. Fluid Mech., 147, 133

Brown, M. R., Canfield, R. C., \& Pevtsov, A. A., Editors, 1999, Magnetic Helicity in Space and Laboratory Plasmas, (Washington, D.C.: AGU)

Canfield, R. C. \& Pevtsov, A. A. 1998, ASP Conf. Ser. 140, 131

Chae, J. 2001, ApJ, 560, L95

Démoulin, P., Mandrini, C. H., van Driel-Gesztelyi, L., Thompson, B., Plunkett, S., Kövári, Z., Aulanier, G., \& Young, A. 2002, A\&A, 382, 650

DeVore, C. R. 2000, ApJ, 539, 944

Grigoryev, V. M. \& Ermakova, L. V. 2002, Solar Phys., 207, 309

Hale, G. E. 1927, Nature, 119, 708

Kusano, K., Maeshiro, T., Yokoyama, T. \& Sakurai, T. 2002, ApJ, 577, 501

Leka, K. D. et al. 1996, ApJ, 462, 547

Linton, M. G., Dahlburg, R. B., Fisher, G. H., \& Longcope, D. W. 1998, ApJ, 507, 404

Longcope, D. W., Fisher, G. H., \& Pevtsov, A. A. 1998, ApJ, 507, 417

Longcope, D. W., Linton, M. G., Pevtsov, A. A., Fisher, G. H., \& Klapper, I. 1999, in Magnetic Helicity in Space and Laboratory Plasmas, ed. M. R. Brown, R. C. Canfield \& A. A. Pevtsov (Washington, D.C.: AGU), 93

Martin, S. F., Bilimoria, R., \& Tracadas, P. W. 1994, in Solar Surface Magnetism, ed. R. J. Rutten \& C. J. Schrijver, (Dordrecht: Kluwer), 303

Martin, S. F. \& McAllister, A. H. 1996, in Magnetodynamic Phenomena in the Solar Atmosphere - Prototypes of Stellar Magnetic Activity, ed. Y. Uchida, T.Kosugi

\& H. S. Hudson, (Dordrecht: Kluwer), 497

Mickey, D. L. 1985, Solar Phys., 97, 223

Moon, Y.-J., Chae, J., Choe, G. S., Wang, H., Park, Y. D., Yun, H. S., Yurchyshyn, V., \& Goode, P. R. 2002, ApJ, 574, 1066

Pevtsov, A. A., Canfield, R. C., \& Latushko, S. M. 2001, ApJ, 549, L261

Pevtsov, A. A., Canfield, R. C., \& Metcalf, T. R. 1995, ApJ, 440, L109 
Porties-Fozzani, F. et al. 2001, Solar Phys., 203, 289

Rust, D. M. \& Kumar, A. 1996, ApJ, 464, L199

Sakurai, T. \& Hagino, M. 2003, J. Korean Astron. Soc., 36, S7

Seehafer, N. 1990, Solar Phys., 125, 219

Seehafer, N., Gellert, M. Kuzanyan, K. M., \& Pipin, V. V. 2003, Adv. Space Research, 32,1819

Tian, L., Bao, S., Zhang, H., \& Wang, H. 2001, A\&A, 374, 294

Updike, A. \& Pevtsov, A. A. 2003, ApJ, in preparation 